\title{
LES ADJECTIFS A VALEUR INTENSIVE DANS LES GROUPES NOMINAUX
}

\author{
Pierre-André Buvet \\ laboratoire LDI \\ UMR 7187 CNRS-Université Paris 13
}

La tradition grammaticale distingue au moins deux sortes d'adjectifs : les déterminatifs et les qualificatifs, $c f$. Grevisse 1980. Les premiers sont des prédéterminants, c'est-à-dire qu'ils participent à l'actualisation des substantifs en saturant la position frontale d'un groupe nominal, par ex. plusieurs dans Il a pris plusieurs photos, $c f$. Leeman 2004. Ce premier type d'adjectifs n'est pas pris en compte dans cette étude. Les seconds ne sont pas sans rapport avec la détermination car, en position épithète, il s'agit soit de modifieurs, par ex. beau dans Il a acheté un beau tapis, soit de constituants d'une détermination figée, par ex. bleu dans Il a une peur bleue de parler en public, $c f$. Buvet sous-presse et Noailly 1999. De nombreux adjectifs qualificatifs sont également compatibles avec la position attribut, $c f$. Riegel 1982. La position attribut d'un adjectif et sa compatibilité avec la pronominalisation le dans une construction en être sont les deux principaux critères retenus pour faire état du fonctionnement prédicatif des adjectifs, $c f$. Picabia 1978 : Son tapis est beau mais le tien l'est tout autant.

Les adjectifs se caractérisent par leur extrême hétérogénéité syntactico-sémantique, cf. Gross \& Lim 1996 : (i) Il a utilisé un tissu doux; (ii) Le chemin a une pente douce ; (iii) C'est un doux rêveur. L'emploi (i) de doux se combine avec toutes sortes de noms lorsqu'il spécifie une consistance tactile (une matière douce, un papier doux, une peau douce, etc.) ; la séquence tissu doux est libre ${ }^{1}$. L'emploi (ii) implique une relation collocationnelle entre le nom et l'adjectif (la valeur intensive de doux procède de sa combinatoire avec le seul nom pente, c'est-à-dire qu'elle n'est pas attestée avec des synonymes du substantif comme déclivité et inclinaison); il s'agit d'un semi-phrasème ${ }^{2}$. L'emploi (iii) est le constituant d'une séquence figée (doux rêveur est sémantiquement équivalent à idéaliste) ; c'est un phrasème ${ }^{3}$.

La fonction lexicale ANTIMAGN rend compte de la relation entre le collocatif doux et le nom pente tandis que la fonction lexicale MAGN fait état de la relation de ce même nom avec le collocatif abrupte, C'est une pente abrupte, $c f$. Mel'cŭk et Alii 1999. La première fonction lexicale décrit les collocatifs dont la valeur est l'intensité faible, le bas degré. La seconde fonction lexicale rend compte des collocatifs dont la valeur est l'intensité forte, le haut degré. L'intensité peut être spécifiée soit directement par une unité lexicale, par ex. la valeur d'adorer est celle d'apprécier combinée à une intensité forte dans Il adore ce film, soit par une combinatoire faisant appel à des marqueurs spécifiques, dits marqueurs de l'intensité. Ces marqueurs concernent exclusivement des prédicats graduables ${ }^{4}$ comme le prouve la combinatoire non systématique de l'adverbe intensif très avec des adjectifs prédicatifs, cf. Goes 1999: Il est $(E+$ très $)$ intelligent; Il est (E+*très) manchot.

La forme des marqueurs de l'intensité dépend des emplois prédicatifs avec lesquels ils se combinent. Les adjectifs prédicatifs et les verbes prédicatifs sont surtout compatibles avec des adverbes, par ex. Il apprécie énormément ce film ou Il est extrêmement ambitieux. Les noms prédicatifs sont compatibles avec des prédéterminants, par ex. beaucoup de dans Il a beaucoup de fièvre, des verbes supports, par ex. brûler dans Il brûle d'impatience de te le dire, des modifieurs propositionnels, par ex. à revendre dans $I l$ a du courage à revendre, et des adjectifs, herculéen dans Il a une force herculéenne, cf. Buvet 2001, Buvet \& Alii 1995, Daladier 1996 et Romero 2001 et 2005.

Nous présentons les marqueurs de l'intensité qui correspondent à des adjectifs monolexicaux ou polylexicaux. Nous distinguons ces adjectifs selon qu'il s'agit de modifieurs libres, de modifieurs liés et 
de constituants d'une détermination figée. Nous précisons quelles sont leurs conditions d'occurrences dans les groupes nominaux en nous appuyant sur les descriptions des adjectifs et des déterminants du français qui proviennent de deux bases de données exhaustives.

\section{Les modifieurs libres de nature adjectivale}

Après avoir défini la notion de modifieur libre, nous discutons des adjectifs qui sont des modifieurs libres puis nous nous intéressons plus particulièrement à ceux qui ont une valeur intensive.

\subsection{A propos des modifieurs libres}

Les modifieurs analysés comme de simples expansions des noms sont rattachés aux substantifs-têtes indépendamment des prédéterminants et, de ce fait, sont exclus du champ d'étude de la détermination ${ }^{5}$. Nous avons opté pour une autre analyse, selon laquelle la détermination d'un nom résulte de tous les constituants du groupe nominal qui ne sont pas le substantif-tête. Aussi, les modifieurs sont définis comme des constituants déterminatifs dont le mode de fonctionnement est explicable à partir de l'opposition 'détermination prédicative' vs 'détermination argumentale', $c f$. Buvet sous presse.

Les modifieurs ont les deux caractéristiques suivantes: leurs occurrences sont facultatives et ils sont généralement postposés aux têtes nominales ${ }^{6}$. Les modifieurs libres ont une caractéristique supplémentaire: les occurrences des prédéterminants, quels qu'ils soient, ne dépendent pas de leurs propres occurrences : J'aime son style (E + flamboyant).

Nous distinguons les modifieurs selon que les têtes nominales fonctionnent comme des prédicats ou bien comme des arguments, c'est-à-dire selon qu'ils sont constitutifs d'une détermination prédicative ou d'une détermination argumentale. Fondamentalement, la détermination prédicative est celle des prédicats nominaux, c'est-à-dire des substantifs qui correspondent au noyau prédicatif dans une construction à support, et la détermination argumentale celle des substantifs en position sujet ou complément relativement à un emploi prédicatif donné. La distinction entre les deux sortes de détermination permet d'expliquer, entre autres, la valeur quantitative et aspectuelle du modifieur adjectival dans Il a fait de nombreuses bêtises (détermination prédicative) et sa seule valeur uniquement quantitative dans Il a choqué de nombreuses personnes (détermination argumentale).

L'incorporation d'un contenu propositionnel dans un autre contenu propositionnel s'effectue notamment par le biais d'un groupe nominal: Ses nombreuses bêtises ont choqué de nombreuses personnes. Les valeurs du modifieur nombreuses ne sont pas altérées par le fait que le nom bêtises fonctionne ici comme un argument, c'est-à-dire qu'il n'est plus le noyau prédicatif de l'énoncé. Autrement dit, les propriétés du modifieur du nom dans la détermination prédicative sont conservées dans la détermination argumentale. En conséquence, pour étudier les faits de détermination, nous distinguons les substantifs selon qu'ils peuvent non être des prédicats ${ }^{7}$. Nous établissons l'intérêt de cette distinction lorsque les modifieurs sont des adjectifs.

\subsection{Remarques sur les modifieurs adjectivaux}

En français, la catégorie des adjectifs se justifie principalement à partir de deux propriétés conjointes des adjectifs monolexicaux. La première propriété est d'ordre morphologique : le même système flexionnel sert à marquer les variations en genre et en nombre des adjectifs et des substantifs. La seconde est d'ordre syntaxique : les occurrences des adjectifs dépendent de celles des noms alors que l'inverse est souvent inexact; un mur $(E+$ fragile $)$; un $\left({ }^{*} E+\right.$ mur $)$ fragile ${ }^{8}$. Cette justification ne convient pas aux adjectifs polylexicaux car ils peuvent être invariables ${ }^{9}$ et leur forme ne permet pas toujours de les dissocier des compléments de noms; les modifieurs ont la même structure de surface dans Le mur d'un immeuble 
désaffecté et Le mur d'une qualité irréprochable mais d'un immeuble désaffecté est un complément du nom et d'une qualité irréprochable un adjectif polylexical en position épithète. La position attribut permet de différencier les compléments de noms des modifieurs adjectivaux : *Le mur est d'un immeuble désaffecté ; Le mur est d'une qualité irréprochable. La comptabilité avec les positions attribut et épithète et la pronominalisation en le dans une construction en être permet de définir les adjectifs polylexicaux comme tels, cf. Gross 1996 : Un citoyen en pétard; Ce citoyen est en pétard et cet autre l'est aussi. Y contribuent également la possibilité qu'ils permutent dans l'une ou l'autre position avec des adjectifs monolexicaux, par ex. Une chemise (jaune canari + verte) et La chemise est (jaune canari + verte), ainsi que la coordination avec des adjectifs simples, par ex. Une histoire amusante et haute en couleurs et Une personne agréable mais un peu soupe-au-lait. Des adjectifs monolexicaux et polylexicaux autorisent la combinatoire avec des adverbes ${ }^{10}$, par ex. Une fille véritablement jolie et Un garçon véritablement mal embouché, ainsi que le superlatif, par ex. La plus jolie fille et Le garçon le plus mal embouché.

Aucune des propriétés mentionnées ci-dessus ne peut caractériser un adjectif lorsqu'elle est considérée isolément, mais la conjonction de plusieurs le permet. Les propriétés qui justifient la catégorisation des adjectifs monolexicaux ne recoupent pas exactement celles qui contribuent à la catégorisation des adjectifs polylexicaux ${ }^{11}$.

D'autres propriétés permettent de distinguer et de classer les adjectifs polylexicaux, $c f$. Buvet 2008. Une première typologie est fondée sur la nature et le mode d'agencement de leurs constituants : (i-a) les séquences figées formées à partir d'un adjectif en position initiale, par ex. amer comme chicotin; (i-b) les séquences figées comportant un adjectif en position finale, par ex. hautement qualifié ; (i-c) des groupes prépositionnels, par. ex. dans l'embarras; (i-d) tous les autres cas de figure. Une seconde typologie prend en compte leur caractère totalement ou partiellement figé, $c f$. Mejri 1997: (ii-a) les séquences dont les éléments sont tous dans la portée du figement, par ex. agité du bocal; (ii-b) les séquences telles que l'adjectif en position initiale est hors de la portée du figement, par ex. volage comme un papillon; (ii-c) les séquences telles que l'adjectif en position finale est hors de la portée du figement, par ex. à moitié endormi ; (ii-d) les autres cas de figure, par ex. bien placé pour le savoir.

L'analyse de la structure interne des adjectifs polylexicaux des types (i-b) et (ii-b) ou des types (i-c) et (iic) montre que les éléments dans la portée du figement sont parfois des marqueurs de l'intensité des éléments hors de la porté du figement en position initiale, par ex. bête comme ses pieds, ou finale, par ex. bon dernier. Considérés globalement, ils n'expriment pas le bas et le haut degré. Ce sont les adjectifs polylexicaux des types (i-c) et (ii-a) ou (i-d) et (ii-a) qui sont des marqueurs intensifs des noms prédicatifs, $c f$. infra.

Certains adjectifs monolexicaux sont non-compatibles avec la position attribut; ce qui les différencie fondamentalement des adjectifs polylexicaux. Les adjectifs monolexicaux qui acceptent uniquement la position épithète sont soit des adjectifs relationnels, $c f$. Gross \& LiM 1996, La crise $(E+* e s t)$ pétrolière, soit des constituants de noms composés, $c f$. Mathieu-Colas 1996, Le trou (E+*est) noir, soit des constituants d'une détermination figée. Seul le dernier cas de figure est concerné par l'expression du bas ou du haut degré, $c f$. infra.

\subsection{Les adjectifs à valeur intensive}

Les adjectifs, les noms et les verbes prédicatifs caractérisés par des marqueurs de l'intensité sont du type état, par ex. Il est très triste, Il éprouve beaucoup de tristesse et Cela l'attriste énormément, ou bien du type événement, par ex. Il pleut énormément et Il y a un violent orage. Les différents emplois prédicatifs, quelle que soit leur catégorie grammaticale, sont susceptibles d'une graduation telle que l'intensité spécifiée est bipolaire ; il peut s'agir du haut degré, l'intensité forte, par ex. Il l'a beaucoup apprécié, ou du bas degré, l'intensité faible, par ex. Il l'a peu apprécié. 
Plus de 1000 adjectifs monolexicaux fonctionnent comme des marqueurs de l'intensité forte ou faible de noms prédicatifs ${ }^{12}$. Ils se caractérisent par d'importantes contraintes distributionnelles, au point que certains ont un caractère collocatif très marqué, $c f$. Blumenthal \& Hausman 2006. Trois paramètres permettent de les classer : leur signification, leur construction et leur distribution. Le premier paramètre concerne directement l'expression du haut ou du bas degré ; il permet de distinguer les adjectifs qui sont plutôt sémantiquement transparents, par ex. forte dans une voix forte, des adjectifs qui ne le sont pas, par ex. royale dans Une magnificence royale. Le deuxième paramètre conduit à différencier les adjectifs monovalents, par ex. claironnante dans une voix claironnante, des adjectifs bivalents, par ex. doué dans Un garçon doué de ténacité. Le troisième paramètre consiste à distinguer les adjectifs qui ont une distribution réduite, par ex. léger dans Une (douleur + poussée + nuance) légère de ceux qui sont des collatifs, par ex. impardonnable dans Un crime impardonnable. Il s'ensuit la mise en évidence de quatre classes d'adjectifs simples.

La première classe regroupe des adjectifs monovalents et distributionnellement contraints qui ont une signification plutôt transparente. Ce ne sont pas des collocatifs dans la mesure où : (i) ils caractérisent des substantifs sémantiquement hétérogènes; (ii) leur valeur intensive ne procède pas de leur combinatoire. La classe comporte soit des marqueurs de l'intensité forte comme immense dans Un immense (effort + potentiel + succès $+\ldots)$, soit des marqueurs de l'intensité faible comme faible dans Une faible (brise + nombre + quantité + ....).

La seconde classe rassemble des adjectifs monovalents et distributionnellement contraints qui ont une signification plutôt opaque. Ils tendent à être des collocatifs du fait que leur valeur intensive résulte de leur combinatoire, mais ils ne le sont pas au sens strict du terme car les substantifs qu'ils caractérisent ne sont pas nécessairement apparentés sur le plan sémantique. Ils expriment soit le haut degré comme invincible dans Un (argument + difficulté + obstacle) invincible soit le bas degré comme menu dans Un(e) menu(e) (bruit + soin + tâche).

La troisième classe est constituée d'adjectifs monovalents qui sont des collocatifs tant sur le plan distributionnel que sur le plan sémantique. Leur signification n'est pas transparente et un très petit nombre de substantifs, voire un seul, les acceptent comme modifieurs. Ce sont soit des marqueurs de l'intensité forte comme inexpiable dans Un(e) (crime + faute) inexpiable ou insondable dans Un mystère insondable soit des marqueurs de l'intensité faible comme passager dans Une averse passagère.

La quatrième comporte tous les adjectifs bivalents de la liste. Ils ont une double particularité : (i) ils contribuent à instancier la relation entre un prédicat nominal et un argument humain ; (ii) ils expriment uniquement le haut degré du prédicat par ex. cloué dans Un voyageur cloué de (peur + stupeur) ou pâle dans Un client pâle de (colère + terreur).

Une cinquantaine d'adjectifs polylexicaux constituent une cinquième classe, par ex. à faire rougir un saint dans Une beauté à faire rougir un saint ou tout-puissant dans un chef tout-puissant. Leur distribution est parfois diversifiée, par ex. du tonnerre dans un(e) (ambiance +bruit + défi ...) du tonnerre mais le plus souvent restreinte au point qu'il s'agit de collocatif, par ex. en béton dans Un alibi en béton. Remarquons que la plupart des adjectifs polylexicaux à valeur intensive sont sémantiquement opaques.

Lorsque les marqueurs de l'intensité sont des adjectifs, monolexicaux et polylexicaux, ils caractérisent des noms prédicatifs potentiellement graduables. L'expression du haut degré ou du bas degré implique alors deux situations selon le caractère plus ou moins subjectif de l'information transmise. Les adjectifs se contentent de dénoter l'intensité dans un cas, par ex. Une foule (compacte + dense + importante) a assisté au concert tandis que, dans l'autre cas, les adjectifs dénotent l'intensité et, par ailleurs, font état de l'implication du locuteur dans son propos, par ex. Une foule (considérable + gigantesque + impressionnante + innombrable) a assisté au concert. Les adjectifs sémantiquement opaques relèvent presque systématiquement du deuxième cas de figure, indépendamment de leur caractère plus ou moins 
collocatif; leur valeur est l'intensité forte ou faible et, parallèlement, ils ont une dimension subjective indéniable, par ex. Un(e) (refus + pluie) cinglant(e) et Un(e) (faute + larcin) véniel(le $)^{13}$.

Cet aspect énonciatif de l'expression du haut ou du bas degré est encore plus marqué avec les adjectifs à valeur intensive qui sont des modifieurs liés ou à des constituants d'une détermination figée.

\section{Les modifieurs liés de nature adjectivale}

Les modifieurs liés sont soit des modifieurs adjectivaux, par ex. polaire dans il fait un froid polaire soit des modifieurs propositionnels du type complétive, par ex. que le climat a empiré dans Il a l'impression que le climat a empiré et du type infinitive, par ex. de le croire dans Il a la naïveté de le croire. Il est question ici principalement d'adjectifs monolexicaux. Après avoir défini la notion de modifieur lié, nous l'examinons du point de vue de la prédication seconde puis nous précisons en quoi les adjectifs dits attributs de l'objet participent à l'expression du haut degré.

\subsection{A propos des modifieurs liés}

Les modifieurs se distinguent des prédéterminants du fait que les occurrences des premiers dépendent de celles des seconds alors que l'inverse est inexact, $c f$. supra. Cependant, les modifieurs peuvent influer sur la nature des prédéterminants. En l'absence de modifieur, frousse se combine avec l'article défini plutôt qu'avec l'article indéfini dans Il a (la + ?une) frousse. En présence d'un modifieur, l'article défini est exclu alors que l'article indéfini est obligatoire dans $\mathrm{Il}$ a (*la + une) frousse qui le paralyse.

Le caractère plus ou moins figé d'une détermination constituée obligatoirement d'un prédéterminant et d'une expansion conduit à distinguer deux situations : (i) Il a une peur qui remonte à loin; (ii) Il a une peur de tous les diables. En l'absence d'une expansion du nom, l'article zéro est le seul prédéterminant admis par le substantif peur dans une construction en avoir, cf. Anscombre 1991: Il a $(E+*$ de la $+* l a+$ *une) peur. Par contre, en présence d'une expansion, l'article zéro est exclu et c'est l'article indéfini qui est exigé : Il a $\left(^{*} E+\right.$ une) peur qui remonte à loin; Il a $\left(^{*} E+\right.$ une) peur de tous les diables ${ }^{14}$. Au-delà de cette propriété commune, l'expansion de (i) et celle de (ii) ne sont pas comparables car seule la première est analysable du point de vue de sa relation avec peur: La peur qu'il a remonte à loin; *La peur qu'il a est de tous les diables. Le fait que la détermination de peur est moins figée dans (i) que dans (ii) conduit à distinguer qui remonte à loin de de tous les diables; la première expansion correspond à un modifieur lié, la seconde à un constituant de détermination figée, $c f$. infra.

Les modifieurs liés de nature adjectivale sont des constituants déterminatifs facultatifs tels que leurs occurrences contraignent la nature des prédéterminants dont ils dépendent. La seconde propriété les différencie des modifieurs libres qui sont aussi des constituants déterminatifs facultatifs mais tels que leurs occurrences sont sans influence sur celles des prédéterminants. Un même adjectif à valeur intensive peut être un modifieur libre ou lié selon le substantif auquel il se rapporte : forte dans Il a exercé une ( $E+$ forte) poussée sur le mur et Il a eu une (*E + forte) fièvre ou carabinée dans Il a reçu une gifle (E+ carabinée) et Il a une fièvre (*E + carabinée) ; la première occurrence de forte et carabinée est un modifieur libre, la seconde occurrence un modifieur lié. Les modifieurs liés, comme les modifieurs libres, sont des adjectifs compatibles avec la position attribut : La poussée qu'il a exercée sur le mur était forte; La fièvre qu'il a eue était forte; La claque qu'il a reçue était carabinée; La fièvre qu'il a eue était carabinée.

Il y a des marqueurs de l'intensité qui sont uniquement des modifieurs liés : glacial dans Il fait un froid ( $E$ + glacial). Ces modifieurs liés sont également des adjectifs compatibles avec la position attribut, Le froid est glacial, alors que les constituants adjectivaux d'une détermination figée ne le sont pas. Ainsi, l'adjectif aveugle lorsqu'il se combine avec le nom confiance dans une construction en avoir correspond à 
un constituant d'une détermination figée parce que, d'une part, il impose l'article indéfini comme prédéterminant et, d'autre part, il a uniquement un statut déterminatif, cf. infra: Il a $(E+*$ une) confiance en elle; Il a une confiance (*E + aveugle) en elle; *Sa confiance en elle est aveugle.

Les faits de langue présentés ci-dessus relèvent de la détermination prédicative dans la mesure où les modifieurs liés s'observent uniquement avec des prédicats nominaux, c'est-à-dire dans des constructions à support, $c f$. Gross et Vives 1986. Les restrictions qui affectent les prédéterminants sont imputables aux propriétés des substantifs; il s'agit notamment de noms prédicatifs actualisés par le verbe support avoir: Il $a(* E+d u+* l e+* u n)$ courage ; Il a $\left({ }^{*} E+*\right.$ de la $\left.+* l a+* u n e\right)$ faim ; Il a (*E $+*$ de la + la + une cote). L'adjonction d'un adjectif à valeur intensive au nom impose l'article indéfini : Il $a\left({ }^{*} E+* d u+* l e\right.$ + un) courage extraordinaire; Il a $\left(^{*} E+{ }^{*} d e l a+* l a+\right.$ une) faim inépuisable; Il a $(E+* d e ~ l a+* l a+$ une cote) incroyable.

Remarquons que les modifieurs liés sont parfois des adjectifs polylexicaux : Il lui a apporté (*son + un) soutien sans réserve; Il a manifesté (*de l'+ *l'+ *son + un) optimisme de commande; Il a un culot à faire pâlir un mort.

Les adjectifs impliqués dans une prédication participent également à l'expression du haut et du bas degré.

\subsection{Adjectifs et prédication seconde}

Les adjectifs de la prédication seconde ont les caractéristiques des modifieurs liés bien que leur mode de rattachement aux substantifs-têtes soit spécifique. Ils se distinguent des modifieurs libres par leur caractère obligatoire dans une construction en avoir, entre autres constructions : (i) Il a le nez (*E+ rouge) ; (ii) Il a le livre (E + rouge). L'adjectif rouge est : (i) attribut de l'objet dans le premier énoncé car il «forme le troisième constituant du syntagme verbal et est distinct du C.O.D. »; (ii) épithète dans le second énoncé car il « fait partie du C.O.D au même titre qu'une expansion prépositionnelle ou relative » Riegel 1988. Le rattachement de l'adjectif au nom est moins marqué en tant que modifieur lié qu'en tant que modifieur libre comme l'atteste la pronominalisation du complément : nez peut être représenté par $l$ ' indépendamment de rouge dans le premier énoncé, Il l'a rouge, tandis que livre ne peut pas l'être sans rouge dans le second, Il l'a. La négation contribue aussi à différencier le mode de fonctionnement de l'adjectif rouge car il y a une seule interprétation dans Il n'a pas le nez rouge (c'est la couleur du nez qui est niée) tandis que deux interprétations sont possibles dans Il n'a pas le livre rouge (soit il n'y a aucun livre, soit il y en a mais aucun de rouge). Le caractère inaliénable de la relation entre l'humain représenté par $i l$ et la partie du corps spécifiée par nez et le caractère contingent de la relation entre l'humain représenté par $i l$ et l'artefact spécifié par livre expliquent les phénomènes mentionnés ci-dessus à propos de ce qui distingue les deux énoncés. Selon Furukawa 1996, en cas de prédication seconde, le verbe avoir devient « une sorte d'adhésif qui colle le SN1 et le SN2 en se dispensant, pour une large part, de son rôle fondamental [... bien qu'il] n'en garde pas moins le sens de possession ». La nature des adjectifs correspondant à des attributs de l'objet est un paramètre fondamental.

Selon Riegel 1988, seuls les adjectifs dénotant « une propriété inhérente (essentielle)» du substantif postverbal sont autorisés alors que ceux qui s'interprètent « comme un état transitoire (accidentel) » sont exclus. Toutefois, si l'on admet que bleu est un adjectif du type essentiel et que irrité un adjectif du type transitoire, on observe qu'il s'agit tous les deux de modifieurs liés dans Il a les yeux (*E + bleus) ; Il a les yeux $\left({ }^{*} E+\right.$ irrités $)$. Les modifieurs liés sont des adjectifs qui contribuent à singulariser les noms auxquels ils se rapportent. L'article défini y contribue du fait de sa « valeur quantificatrice exhaustive » Ibidem. Autrement dit, le prédéterminant accentue la spécificité de la relation entre le nom et l'adjectif.

D'une manière générale, la relation partie-tout entre le sujet et le complément explique le caractère obligatoire des modifieurs liés. Les occurrences de l'article défini peuvent être imputables à un effet discursif : c'est un choix du locuteur de renforcer avec ce prédéterminant la spécificité de la relation entre 
l'adjectif et le nom. Cependant, lorsque les adjectifs sont du type transitoire, l'article indéfini n'est pas possible : Il a (les $+*$ des) yeux irrités. De ce point de vue, l'opposition entre les adjectifs du type essentiel et les adjectifs du type transitoire conserve sa pertinence pour l'analyse des adjectifs correspondant à des attributs de l'objet.

L'effet discursif qu'implique la prédication seconde concerne non seulement les noms élémentaires mais aussi les noms prédicatifs.

\subsection{Intensité et prédication seconde}

Deux sortes de noms prédicatifs sont compatibles avec des adjectifs dans une prédication seconde : les substantifs relatifs à une qualité ou une faculté humaine, Il a l'intelligence claire, et les substantifs en rapport avec toutes sortes de processus, Les pieds noirs ont le vote sale ${ }^{15}$. Dans les deux cas de figure, la prédication seconde s'observe dans une construction en avoir telle que la position sujet est saturée par un groupe nominal ou un pronom en rapport avec un humain ${ }^{16}$. Le même procédé discursif est à l'œuvre, à savoir la focalisation sur le modifieur lié met en avant la valeur de l'adjectif dans la proposition comme l'atteste les exemples authentiques suivants: Il a la démarche souple; Il a l'esprit vif; Il a le remords cruel; Il a la passion dévorante.

Parmi les substantifs relatifs à une qualité ou à une faculté humaine, on doit distinguer ceux qui dénotent une propriété intrinsèque du type essentiel (démarche ou esprit) de ceux qui dénotent une propriété intrinsèque du type accidentel (remords ou passion), $c f$. Anscombre 2003. À l'instar du méronyme nez, démarche et esprit sont inhérents à tous les humains alors que remords ou passion ne les caractérisent pas tous d'une façon nécessaire et permanente. Du point de vue de la prédication seconde, cette distinction se manifeste comme suit : (i) les adjectifs sont plus variés avec les substantifs de la catégorie 'intrinsèqueessentiel', par ex. Il a la démarche (souple+raide+hésitante+bringuebalante) et Il a l'esprit (vif+lent+étroit+large), qu'avec les substantifs de la catégorie 'intrinsèque-accidentel', par ex. Il a le remords (cruel $+*$ méchant + ?tardif + ?sincère) et Il a la passion (dévorante + ?romanesque $+*$ torride + *intense) ; (ii) le remplacement de l'article défini par l'article indéfini est plus facile avec la première catégorie de noms, par ex. Il a (la +une) démarche souple et Il a $\left(l^{\prime}+\right.$ un) esprit vif, qu'avec le seconde, par ex. Il a (le $+*$ un) remords cruel et Il a (la + ?une) passion dévorante.

La détermination des noms de la catégorie 'intrinsèque-essentiel' apparaît donc moins contrainte que celle des noms de la catégorie 'intrinsèque-accidentel' dans la prédication seconde. Ce qui conduit à rapprocher la première catégorie des noms de la catégorie des méronymes, indépendamment du fait qu'il s'agisse de noms prédicatifs dans un cas et de noms élémentaires dans l'autre. Il est alors remarquable que l'expression du haut degré est principalement imputable à des adjectifs constitutifs de la détermination la plus contrainte : Il a la joie explosive, Il a la franchise brutale, Il a la rancune tenace, Il a la détente facile, etc. Les adjectifs à valeur intensive de la détermination la moins contrainte sont généralement polylexicaux : Il a le moral au beau fixe; Il a un cour gros comme ça.

Les noms prédicatifs en rapport avec toutes sortes de processus impliqués dans une prédication seconde ont également une détermination contrainte, $c f$. Gaatone 1991, Riegel 1988 et Kupferman 2000. Une partie des exemples recensés attestent d'adjectifs qui expriment le haut degré relativement aux noms prédicatifs dont ils dépendent : Il a le pourboire large, Il a le redressement féroce, Il a la taloche lourde, etc. Les exemples de prédication seconde ont tous comme particularité de mettre en avant la manière dont s'effectue le processus plutôt que le processus lui-même. La différence entre Ils votent salement et Ils ont le vote sale est de nature discursive : la construction en avoir permet au locuteur de focaliser sur la façon de voter au détriment du fait de voter, $c f$. Riegel 1996. On l'observe également lorsque le processus est caractérisé en termes de haut degré. La prédication seconde renforce la valeur intensive spécifiée : la séquence adverbiale à valeur intensive dans Ils font des surveillances d'une façon serrée est reprise sous 
une forme adjectivale dans Ils ont la surveillance serrée de telle sorte qu'il y a amplification du haut degré exprimé.

Le renforcement de l'intensité dans une prédication seconde s'observe également lorsque le nom prédicatif dénote une qualité ou une faculté humaine. L'intensité forte spécifiée par idéale dans Elle a la beauté idéale est accrue du fait que l'adjectif fonctionne comme un attribut de l'objet. Le recours à la prédication seconde pour exprimer le haut degré corrobore que les marqueurs de l'intensité peuvent être aussi des marqueurs de la subjectivité lorsqu'ils connotent la façon dont le locuteur prend en charge l'énoncé.

\section{Les constituants adjectivaux d'une détermination figée}

Nous terminons par une présentation rapide des séquences déterminatives figées qui correspondent à des marqueurs de l'intensité.

En premier lieu, les séquences déterminatives figées, par ex. un_noir dans Il a un cafard noir, sont formées de deux constituants déterminatifs : un prédéterminant qui, de ce fait, est en position frontale dans le groupe nominal, et un adjectif qui est positionné, en règle générale, à droite du substantif-tête ${ }^{17}$. Cette configuration est identique à celle des séquences déterminatives non figées formées d'un prédéterminant et d'un adjectif correspondant soit à un modifieur libre, par Il a acheté un livre noir, soit à un modifieur lié, Il a la peau noire. Cependant le constituant adjectival des séquences figées les distinguent des séquences non figées: il s'agit obligatoirement d'un adjectif monolexical car leur catégorisation grammaticale procède d'un autre emploi. Ainsi, bleu dans Il a une peur bleue de sauter est catégorisé comme un adjectif parce qu'un autre emploi de ce mot en a les caractéristiques, comme dans Le ciel est bleu.

En second lieu, les séquences déterminatives figées sont telles que la nature du prédéterminant est corrélée à celle de l'adjectif, par ex. Il éprouve $(* d e l$ ' $+* l$ ' + un) amour fou. Cette propriété est partagée par les séquences déterminatives formées d'un modifieur lié, par ex. Il éprouve (*de l' +*l' + un) amour intense. Le parallèle est néanmoins limité à cette propriété car, dans le premier cas de figure, le constituant adjectival interdit la position attribut, *L'amour qu'il éprouve est fou, alors que le modifieur lié l'autorise, L'amour qu'il éprouve est intense.

En troisième lieu, les séquences déterminatives figées sont très souvent des collocatifs. Du point de vue distributionnel, elles sont autorisées soit par quelques substantifs, sémantiquement apparentés, par ex. bel dans Un bel (abruti + enfoiré + imbécile) soit, le plus souvent, par un seul, par ex. la crue dans Il nous a dit la vérité crue et un artistique dans Ils maintiennent un flou artistique sur cette affaire. Du point de vue sémantique, la séquence déterminative a une signification opaque et sa valeur intensive procède de sa seule combinatoire avec le substantif tête. Seule la combinatoire de une blanche avec colère dans Il est en proie à une colère blanche implique que la séquence déterminative est un marqueur de l'intensité forte; la valeur intensive est exclue avec d'autres substantifs par ex. gelée dans Il y a eu une gelée blanche. Remarquons que des séquences déterminatives formées d'un modifieur libre ont également cette propriété, $c f$. infra, mais qu'elles ne partagent pas d'autres propriétés avec les séquences déterminatives figées.

Les constituants adjectivaux d'une détermination figée ont donc trois points communs avec les modifieurs libres et les modifieurs liés lorsqu'ils participent à l'expression du haut ou du bas degré. Les trois types de conditions d'occurrences des adjectifs à valeur intensive font apparaître un autre point commun, leur caractère idiomatique; il s'explique soit par la nature collocative des constituants adjectivaux et des modifieurs libres soit par la spécificité syntaxique des modifieurs liés, ce sont des attributs de l'objet. Différents travaux ont souligné la dimension énonciative des expressions idiomatiques, $c f$. Langlotz 2006 et Mejri 2002. Pour ce qui est des marqueurs de l'intensité, il s'agirait également de marqueurs de la 
subjectivité lorsqu'il s'agit d'idiotismes. Ce point est à étudier plus en détail. Il serait d'un grand intérêt pour la compréhension automatique de textes car l'identification des marqueurs de l'intensité contribuerait à mettre en évidence le caractère plus ou moins subjectif des discours qui les incorporent.

\section{Bibliographie}

Anscombre, J.-C. (1991). La détermination zéro : quelques propriétés, Langages, 102, 103-124.

Anscombre, J.-C. (2003). Psych-Nouns in French: Semantics and Object Classes, in Language Research special issue, 55-76.

Berthelon, C. (1955). L'expression du haut degré en français contemporain, Romanica Helvetica, 50.

Blumenthal, P. \& Hausman, F. J. (2006). Présentation : collocations, corpus, dictionnaires, Langue française, 150, 313.

Buvet, P.-A. (2008). Quelle description lexicographique du figement pour le TAL ? Le cas des adjectifs à forme complexe, Romanistik 36, 43-54.

Buvet, P.-A. (2001). Les déterminants intensifs, Lingvisticae Investigationes Supplementa, 23, 101-113.

Buvet, P.-A. (sous presse). La dimension lexicale de la détermination en français, Champion : Paris.

Buvet, P.-A. \& Girardin, C. \& Gross, G. \& Groud, C. (1995). Les prédicats d'<affect>, LIDIL, 32, 125-143.

Corblin, F. (1995). Les formes de reprise dans le discours. Anaphores et chaînes de référence, Presses Universitaires de Rennes : Rennes.

Daladier, A. (1996). Le rôle des verbes supports dans un système de conjugaison nominale et l'existence d'une voix nominale en français, Langages, 121, 35-53.

Furukawa, N. (1996). Grammaire de la prédication seconde. Forme, sens et contraintes, Duculot: Louvain-laNeuve.

Gaatone, D. (1991). Un calembour syntaxique en français, Journal of French Language Studies, 1,45-53.

Goes, J. (1999). L'adjectif. Entre nom et verbe, Duculot : Louvain-la-Neuve.

Grevisse, M. (1980). Le bon usage, Duculot : Louvain-la-Neuve.

Gross, G. (1996). Les expressions figées en français : noms composés et autres locutions, Ophrys : Paris-Gap.

Gross G. \& Lim, J.-H. (1996). Une catégorie hétérogène : l'adjectif, Studi Italiani di Linguistica Teoretica e Applicata, XXV:3, 447-460.

Gross, G. \& Vives, R. (1986). Les constructions nominales et l'élaboration d'un lexique-grammaire, Langue française, 69, 5-27.

Kupferman, L. (2000). Avoir et la prédication seconde, Langue française, 127, 67-85.

Langlotz, A. (2006). Idiomatic creativity : a cognitive-linguistic model of idiom-representation and idiom-variation in English, John Benjamins : Amsterdam/Philadelphia.

Leeman, D. (2004). Les déterminants du nom en français. Syntaxe et sémantique, Presses Universitaires de France: 
Paris.

Mathieu-Colas, M. (1996). Essai de typologie des noms composés français, Cahiers de lexicologie, 69, 71-125.

Mejri, S. (1997). Le figement lexical : descriptions linguistiques et structuration sémantique, Faculté des lettres de la Manouba : Tunis.

Mejri, S. (2002). Le figement lexical : nouvelles tendances, Cahiers de lexicologie, 80, 213-225

Mel'čuk, I. \& Clas, A \& Polguère, A. (1999). Dictionnaire explicatif et combinatoire du français contemporain, Les Presses de l’Université de Montréal : Montréal.

Mel'čuk, I. (2003). Collocations dans le dictionnaire, in Les écarts culturels dans les Dictionnaires bilingues, Honoré Champion : Paris, 19-64.

Noailly, M. (1999). L’adjectif en français, Ophrys : Gap-Paris.

Picabia, L. (1978). Les constructions adjectivales. Systématique transformationnelle, Droz: Genève

Riegel, M. (1985). L'adjectif attribut, Presses Universitaires de France : Paris.

Riegel, M. (1988). L'adjectif attribut de l'objet du verbe avoir: amalgame et prédication complexe, Travaux de linguistique, 17, 69-87.

Riegel, M. (1996). Les constructions à élargissement attributif: double prédication et prédicats complexes, in Dépendance et intégration syntaxique : subordination, coordination, connexion, Niemeyer : Tübingen, 189-197

Riegel, M. (1997). Il est gentil de nous avoir aidés ou : A propos de compléments de l'adjectif qui n'en sont pas vraiment ». in Les formes du sens. Études de linguistique française, médiévale et générale offertes à Robert Martin à l'occasion de ses 60 ans, Duculot : Louvain-la-Neuve, 355-365

Riegel, M. \& Pellat, J.-C. \& Rioul R. (1994). Grammaire méthodique du français, Presses Universitaires de France : Paris.

Romero, C. (2001). L'Intensité en français contemporain : analyse sémantique et pragmatique, Thèse de doctorat, Université Paris 8, Saint-Denis.

Romero C. (2005). Les adjectifs intensifs, in L'Adjectif en français et à travers les langues, Caen : Presses Universitaires de Caen, 449-462.

1 «Un syntagme de la langue L est libre si, et seulement si, il peut être construit, à partir d'un contenu informationnel donné, de façon 1) régulière et 2) non contrainte » Mel'cŭk 2003.

2 «Une collocation [est telle que] l'un de ses constituants [...] est choisi librement pour son sens, [...] la base de la collocation [...], l'autre constituant [... ], le collocatif, est sélectionné en fonction du premier » Ibidem.

3 «Un syntagme de L [...qui] ne peut pas être construit, à partir d'un contenu informationnel donné, de façon régulière et non contrainte »Ibidem.

4 Un même prédicat peut être quantifiable et graduable, par ex. explosion dans Il y a eu trois explosions et Il y a une forte explosion. 
5 «A l'intérieur du GN, ces éléments facultatifs [les modifieurs] dépendent du nom, avec lequel ils forment un syntagme plus étendu que le nom, mais plus petit que le GN. Ce constituant intermédiaire sera appelé dorénavant nom expansé » Riegel et alii 1994 : 149.

$6 \mathrm{Il}$ s'agit d'adjectifs, rouge dans $\mathrm{Il}$ a acheté une voiture rouge, de propositions, la relative dans Il a acheté la voiture que je t'avais vendue auparavant, la complétive dans Il avait l'espoir que tout s'arrange au bout du compte, l'infinitive dans Il a eu le courage de dire non, les participiales dans Il a acheté de la viande trop cuite et Il a fait un discours vantant les mérites de notre produit ou de compléments du nom, du salon dans Il n'aime pas les bibelots du salon.

7 Nous appelons noms prédicatifs les premiers et noms élémentaires les seconds.

8 CORBLIN 1995 signale la construction du type LE A. Cependant, les occurrences de cette construction s'observent uniquement dans une situation de deixis ou d'anaphore, par exemple le bleu dans Parmi tous ces pulls, j'aime le bleu.

9 Par exemple, en cours dans une séance en cours. Quelques adjectifs monolexicaux sont parfois invariables, par ex. lilas dans Une robe lilas.

10 Il s'agit d'un critère important pour distinguer les adjectifs simples des noms simples car ces derniers les refusent systématiquement, quelle que soit leur forme.

11 Les formes de la deixis et de l'anaphore distinguent également les adjectifs monolexicaux des adjectifs polylexicaux: Elle a vu des robes dans la vitrine. Elle aime bien (la verte $+{ }^{*}$ celle verte)/Elle aime bien (*la en cuir/celle en cuir).

12 Ils sont tous compatibles aussi bien avec la position épithète qu'avec la position attribut.

13 La position attribut des adjectifs à valeur intensive implique qu'on les analyse aussi comme une prise en charge de l'énoncé par le locuteur, qu'il soit sémantiquement opaque ou transparent, $c f$. Riegel 1997.

14 Lorsque l'article indéfini est également exclu, c'est l'article défini qui est requis : Il a eu $\left(^{*} E+{ }^{*} u n+l a\right)$ peur de sa vie.

15 Exemple de Riegel 1988.

16 Riegel 1988 signale la possibilité d'observer des énoncés tels que la position sujet n'est pas saturé par un argument du type humain ; par exemple, La base loisir a le niveau capricieux.

17 Selon Noailly 1999 : 91, «l'ordre normal du français moderne est l'ordre SA (substantif + adjectif) ». C'est le cas pour tous les adjectifs à deux arguments (un homme libre de préjugés + *un libre de préjugés homme) et de la plupart des adjectifs à un seul argument (sa vie mouvementée + *sa mouvementée vie). L'antéposition de l'adjectif par rapport au nom est souvent le fait «des adjectifs primaires (non dérivés), fréquents et courts »cf. Ibidem : un_beau vase $+*$ un vase beau. 